\title{
Experimental exploration of the upper measuring limit of pressure gauges for ITER by variation of instrumental parameters
}

\author{
Felix Mackel $^{\mathrm{a}}$, Alexey Arkhipov ${ }^{\mathrm{a}}$, Andrea Scarabosio ${ }^{\mathrm{a}}$, Günter Haas ${ }^{\mathrm{a}}$, Jürgen Koll ${ }^{\mathrm{a}}$, Hans Meister ${ }^{\mathrm{a}}$, \\ Fabien Seyvet ${ }^{\mathrm{b}}$, Santiago Terron ${ }^{\mathrm{b}}$ and Philip Andrew ${ }^{\mathrm{c}}$ \\ ${ }^{a}$ Max-Planck-Institut für Plasmaphysik, Boltzmannstraße 2, 85748 Garching, Germany \\ ${ }^{b}$ Fusion for Energy, c/ Josep Pla, n'2, Torres Diagonal Litoral, Edificio B3, 08019 Barcelona, Spain \\ 'ITER Organization, Route de Vinon-sur-Verdon, CS 90 046, 13067 St Paul Lez Durance Cedex, France
}

\begin{abstract}
Hot cathode ionization gauges are intended to be the only in-situ diagnostic for neutral gas pressures in the vacuum vessel of ITER. The development bases on the well established ASDEX pressure gauge. The upper measuring limit for ITER is required to be at least $20 \mathrm{~Pa}$ in hydrogen at a magnetic flux density of up to $8 \mathrm{~T}$. The objective of the presented study is to find parameter and design settings that allow to satisfy this specification. At the same time, the sensitivity shall be high and only weakly dependent on the magnetic field strength. Gauge parameters, specifically the electron emission current, the electrode potentials and the transparency of the acceleration grid, were varied consecutively to assess their impact on the calibration characteristics. The ratio of ion to electron current as a function of pressure and magnetic flux density was obtained for each parameter set.

A monotonic progression of the signal in dependence of the pressure was proven even up to $30 \mathrm{~Pa}$. This was achieved by a low grid transparency and high electric field strength at the cathode. While the former leads to a lower sensitivity, which is unfavorable for measurements in the low pressure range, this can be compensated by a higher electron current.
\end{abstract}

Keywords: Pressure gauge, neutral gas, ionization, diagnostic, ITER

\section{Introduction}

The energy confinement of the core plasma in fusion devices is essentially degraded by the flux of incoming neutral gas atoms at the plasma boundary. In contrast, the presence of a high neutral gas density in the divertor region is desired to enhance the cooling of incoming plasma. Divertor plates are prevented from damage due to a partial conversion of power from the directed particle flux into isotropic radiation originating from electronic transitions in excited atoms and ions [1]. The ITER machine needs thus a good compression ratio of the order of 100 to 1000 to fulfill those demands. Neutral gas pressures in the main chamber as well as in the divertor have to be known to assure a safe and reliable control of the machine. From numerical calculations, the divertor pressure is expected to raise up to $10-15 \mathrm{~Pa}$ during full power operation [2]. The ASDEX-type pressure gauge (APG) is suited to provide local and time resolved measurements in high magnetic fields - it is operated successfully in present-day tokamaks and stellarators [3]. However, the gauge signal was found to saturate at pressures below $20 \mathrm{~Pa}$, which is the upper operational limit as required by ITER [4]. The principle of operation and the physical mechanism leading to signal saturation are described in Section 2. A dedicated experiment as described in Section 3 was set up to show that the requirement on the upper pressure limit in magnetic fields up to $8 \mathrm{~T}$ can be satisfied through minor modifications of design and operational parameters. Experimental results are presented and discussed in Section 4, which is subdivided into three parts treating the different parameter variations. The data is also evaluated with regard to the magnetic field dependence.

\section{Background}

\subsection{Measurement principle of APGs}

The ASDEX type pressure gauge is a hot cathode ionization gauge with a plane-parallel arrangement of electrodes with its axis roughly aligned with the magnetic field. The directly heated filament is thick to withstand Lorentz forces. Fig. 1 shows a sketch of the gauge. The control electrode in front of the filament is biased with a rectangular voltage waveform to chop the electrode current. Spurious DC signals are suppressed effectively by this means. Electrons released by the hot filament are attracted by the acceleration grid and overshoot into the ionization volume. The ions produced here follow the magnetic field lines and finally reach the ion collector, which is on ground potential.

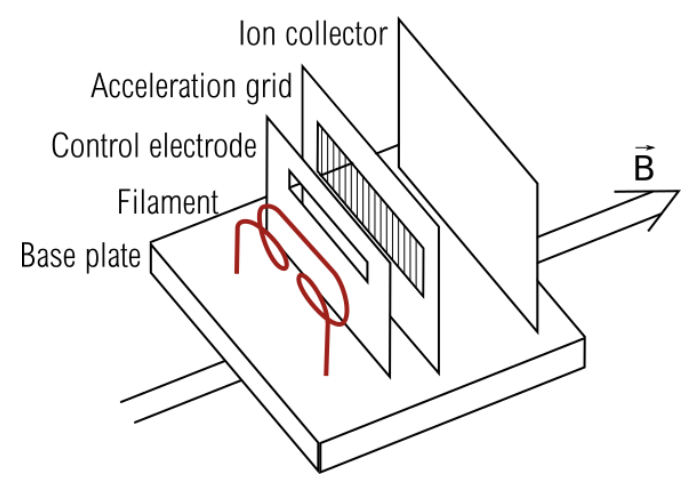

Fig. 1. Schematics of the ASDEX pressure gauge

The electron current reaching the acceleration grid is kept constant via a feedback regulated heating current of the filament. The filament itself has to be biased well 
above ground potential in order to prevent electrons to reach the ion collector. The potential configuration, which is used as a starting point in this investigation, is shown in Fig. 2. It is the outcome of a set of numerical simulations applied to a realistic model that is implemented into a Monte-Carlo code [4]. This separate effort aimed to find potential settings that allow for improving the high-pressure limit.

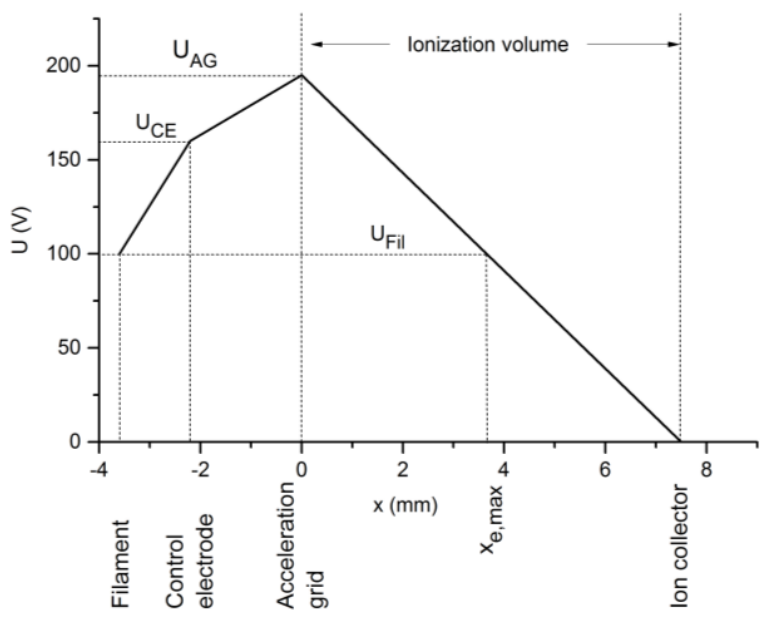

Fig. 2. Reference potential configuration of the electrodes, $\mathrm{x}_{\mathrm{e}, \max }$ designates the reversal point of unscattered electrons.

The base plate and the electrodes are covered with a steel, cubic cap of $2 \mathrm{~cm}$ edge length and an aperture of $8 \mathrm{~mm}$ on the top. The function of the cap is to protect the electrodes from charged particles and fast neutrals from the outside. Since, in steady state, the rate of neutral particles leaving the gauge is equal to that entering the gauge, the flux is a preserved quantity. Therefore, the gauge is actually calibrated for the flux density of neutrals and not the pressure.

\subsection{The saturation effect}

The normalized gauge output is defined as the ratio $I_{i} /\left(I_{e}-I_{i}\right)$, where the ion current $\mathrm{I}_{\mathrm{i}}$ in the denominator accounts for the electrons which originate from ionizations and contribute to the total electron current $\mathrm{I}_{\mathrm{e}}$. Without magnetic field, the gauge output is a linear function of the pressure. This is not the case for $\mathrm{B}>1 \mathrm{~T}$. The sensitivity, which is the ratio of the gauge output and the pressure, increases drastically with the magnetic field strength. The reason for this behavior is found in the dynamics of electrons [3]. At low pressures, the mean free path is long and magnetically guided electrons may return to the filament because they have not lost any of their parallel momentum. This portion is undetected but contributes to the electron density in the ionization volume and is thus statistically relevant. Scattered electrons have not enough energy to overcome the potential well and so they are trapped. The time until they collide with the acceleration grid can be quite long and may take several oscillations until they lose most of their energy in inelastic collisions. This in some sense artificial sensitivity increase gets partially lost at higher pressures when collision probabilities increase significantly. More and more electrons get trapped and contribute to the measured $\mathrm{I}_{\mathrm{e}}$. It is expected that the saturation effect, starting usually around $10 \mathrm{~Pa}$, may be shifted towards higher pressures by different means [5]:

1. Accelerating the electrons faster once they are released. The electric field at the cathode has to be increased. By this means, the probability for an electron to return to the filament is enhanced at high pressures since the cross-section for elastic scattering decreases at higher energies.

2. A reduction of the acceleration grid transparency leads to a larger portion of detected electrons that do not enter the ionization volume. Hence, the sensitivity increase should be attenuated.

3. A reduction of the slit size in the acceleration grid results in a shorter trapping time of the electrons.

4. The higher the electron current, the higher the electron density. This involves increased interactions among the electrons and a higher effective collision rate. Less electrons will then return to the filament.

5. A reduction of the ionization volume leads to less scattering and thus an increased pressure limit.

Except the latter point, the other influences are assessed in the following.

\section{Experimental procedure}

In this test sequence, a prototype gauge head is used that allows exchanging the acceleration grid and the filament. Both parts are bolted, all other components are brazed to the base plate. The prototype is clamped into a mount made of massive copper. Two heating rods are connected to the mount; the temperature of the base plate was kept at $300^{\circ} \mathrm{C} \pm 5^{\circ} \mathrm{C}$ before and during the tests. This prevents changes of the measuring signal due to temperature variations inside or outside the gauge head. Thus, it is well justified that the measured pressure is proportional to the flux density at the gauge position and the flux density is proportional to the gas density inside the gauge. By contrast, the gauge head temperature in ITER will fluctuate due to varying boundary conditions, like e.g. temperature of the mounting structure and heat input by neutrons and gamma radiation. It is foreseen to correct the pressure reading for this influence by postprocessing the measurement signal, taking into account the instantaneous temperature of the base plate, which is deemed to be representative for the gas temperature inside the box. For the investigation presented here, the temperature was kept constant to get a more precise picture of the dependencies.

The pressure was stabilized by a feedback regulated gas inlet valve. The input signal was provided by a Pirani gauge, which remains functional even in high magnetic fields. Two capacitance gauges (Pfeiffer CMR 374) are installed at the main chamber to measure the upstream and downstream pressures. The accuracy of these gauges is well below $1 \%$ in the range from $1 \mathrm{~Pa}$ to $30 \mathrm{~Pa}$. The vacuum vessel is a $1 \mathrm{~m}$ long $10 \mathrm{~cm}$ diameter steel cylinder that intersects the bore of the magnet. A feedback controlled valve injects the gas at one end of 
the tube; the gas is pumped at the other end through an adjustable valve. The magnet provides a maximum magnetic field of $7.9 \mathrm{~T} \pm 0.3 \mathrm{~T}$ (rounded to $8 \mathrm{~T}$ henceforth) for an accessible current of $4.1 \mathrm{kA}$. To acquire the gauge performance in dependence of the magnetic flux density, consecutive pulses have been applied with a flat-top phase of $1.5 \mathrm{~s}$ each. For each parameter setting, measurements have been taken for pressures from $10^{-2}-30 \mathrm{~Pa}$ in hydrogen and magnetic field values from 0 to $8 \mathrm{~T}$. Fig. 3 represents measurements for the baseline configuration with a grid transparency of $40 \%$ and an electron current of $50 \mu \mathrm{A}$. As expected, a linear progression is found in the double logarithmic plot for the case without magnetic field. The sensitivity increases by more than one order of magnitude during the raise of $\mathrm{B}$ up to $1 \mathrm{~T}$ and remains largely unaffected by higher fields. The gap between the $0 \mathrm{~T}$ and the $\mathrm{B} \neq 0 \mathrm{~T}$ curves becomes more narrow with increasing pressure due to the increasing scattering probability. All curves show a monotonic behavior up to $30 \mathrm{~Pa}$ but the one for $8 \mathrm{~T}$ features a considerable flattening at high pressures.

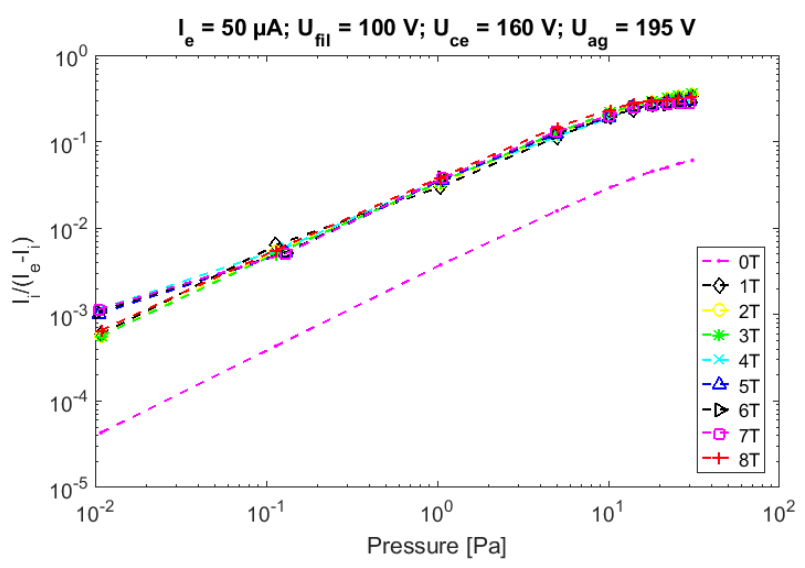

Fig. 3. Normalized gauge output in dependence of pressure and magnetic field as indicated in the legend

\section{Experimental results}

\subsection{Variation of the electron current}

First, the influence of the electron current on the gauge performance at high pressures was tested. The acceleration grid with a transparency of $40 \%$ and a slit width of $0.2 \mathrm{~mm}$ was inserted. The bias of the grids was set to the values as shown in Fig. 2. Electron currents were varied within one order of magnitude from $50 \mu \mathrm{A}$ to $500 \mu \mathrm{A}$. For reasons of clarity, only the progressions for a magnetic flux density of $8 \mathrm{~T}$ are shown in Fig. 4.

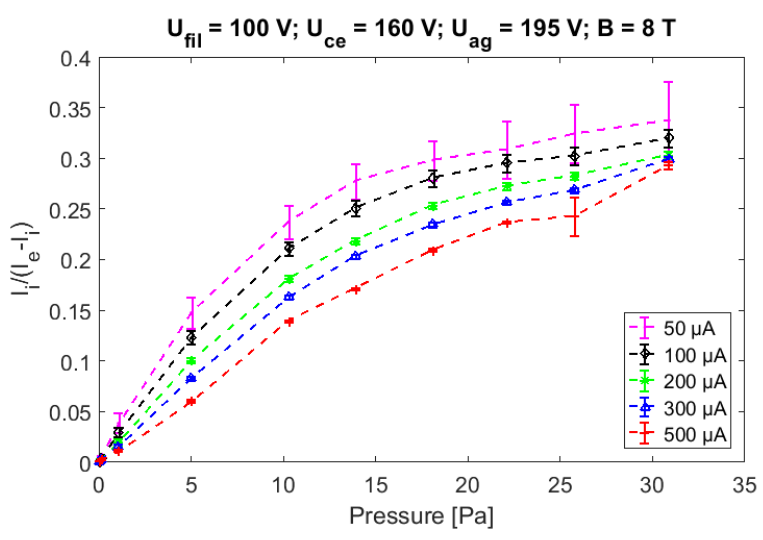

Fig. 4. Gauge output as a function of pressure and electron current for $\mathrm{B}=8 \mathrm{~T}$ and the baseline bias configuration. The error bars are exaggerated by a factor of 10 .

The error bars are derived from the standard deviations of the measuring signals $\mathrm{I}_{\mathrm{e}}$ and $\mathrm{I}_{\mathrm{i}}$ during the flat-top phase of the magnetic field. They are not plotted in the following plots since they are so small.

A monotonic behavior is observed for all electron currents. Increasing the electron current leads to a better linearity of the output as a function of pressure. The slope at high pressures is therefore steepest for $\mathrm{I}_{\mathrm{e}}=500 \mu \mathrm{A}$. This improvement goes to the expense of the independence from the magnitude of the B-field. While for $\mathrm{I}_{\mathrm{e}}=50 \mu \mathrm{A}$, the output is almost not influenced by $\mathrm{B}$, see Fig. 3, there is a considerable dependence for high $I_{e}$ (not presented). Both effects could be explained by the Coulomb interactions of electrons [3]. Elastic scattering leads to more trapped electrons and thus a reduction of the number of electrons that are able to return to the filament. The sensitivity increase is reduced in this way. At the same time, trapped electrons can execute more oscillations at high $\mathrm{B}$ because it inhibits the perpendicular drift motion.

The increase in slope from $26 \mathrm{~Pa}$ to $30 \mathrm{~Pa}$ is not expected and cannot be explained in a phenomenological approach.

\subsection{Variation of the grid potentials}

In a second step, the electrode potentials have been changed successively. The electron current was set to $300 \mu \mathrm{A}$. Fig. 5 shows clearly that the total potential difference influences the sensitivity. With a reduction of the filament potential by $30 \mathrm{~V}$, the ionization rate is drastically enhanced. Stronger biasing of the acceleration grid provokes a further increase of the ion current. In contrast, the increase of the control grid voltage by $20 \mathrm{~V}$ has only a minor effect. 


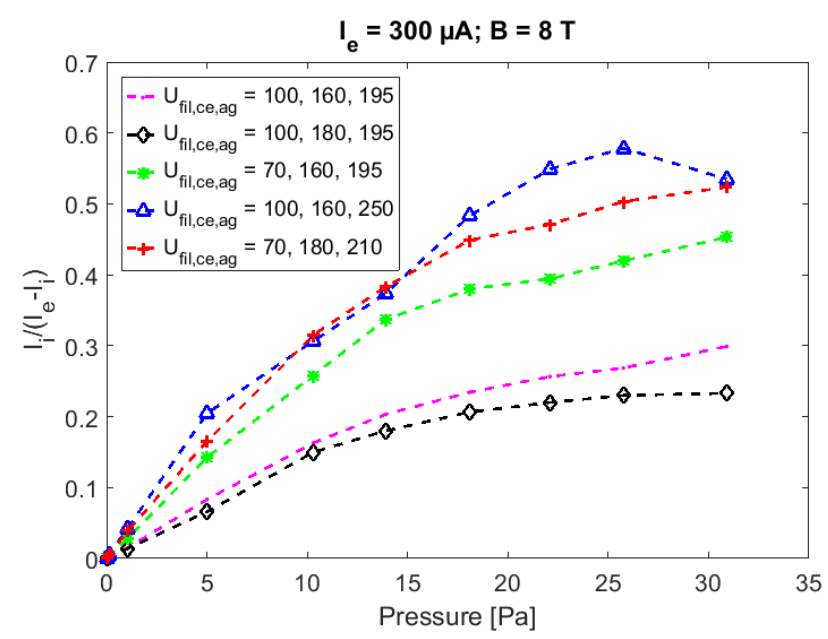

Fig. 5. Gauge performance under variation of electrode potentials as indicated in the legend, in units of volts. The magnetic field is $8 \mathrm{~T}$, the electron current is $300 \mu \mathrm{A}$.

It can be concluded that the electric field in front of the cathode plays a crucial role to extend the upper limit of the measurement range since the control electrode bias was kept high; it was varied in only a limited extent. Also, the relatively high potential difference of $100 \mathrm{~V}$ between the filament and the ion collector may have an influence because it leads to an effective reduction of the ionization volume. Disregarding their finite initial energy, the electrons are able to overcome only half of the distance from the acceleration grid and the ion collector, see $x_{e, \max }$ in Fig. 2. Thus, the chance for a collision decreases compared to a low filament potential. The data is difficult to interpret quantitatively without an adequate numerical tool that also takes Coulomb interactions among electrons into account.

\subsection{Variation of the grid transparency}

Four grids are available with transparencies (tps) of $80 \%, 60 \%, 40 \%$ and $20 \%$ each with the same number of 28 slits, homogeneously distributed on a length of $1.4 \mathrm{~cm}$. This translates into slit widths of $0.4,0.3,0.2$ and $0.1 \mathrm{~mm}$. One additional grid with the doubled number of 56 slits and a tp of $40 \%$ was manufactured to investigate the effect of a reduced slit width while the tp is held constant. The electron current was set to $300 \mu \mathrm{A}$ for all experiments. Results are shown in Fig. 6. The lowest sensitivity is found for the acceleration grid with $20 \%$ tp. The output at low pressures is roughly doubled if the tp is increased to $40 \%$. This ratio becomes slightly lower at high pressures. The sensitivity increases further for $60 \%$ tp but the slope flattens drastically at $\sim 15 \mathrm{~Pa}$. The grid with $80 \%$ tp leads to an unexpected behavior: the sensitivity compared to the $60 \%$ case is lower at low pressures and higher at high pressures with the crossing point at $22 \mathrm{~Pa}$. A reduction of the slit width at $40 \%$ tp resulted in a better performance in terms of sensitivity while the slope at high pressures is comparable.

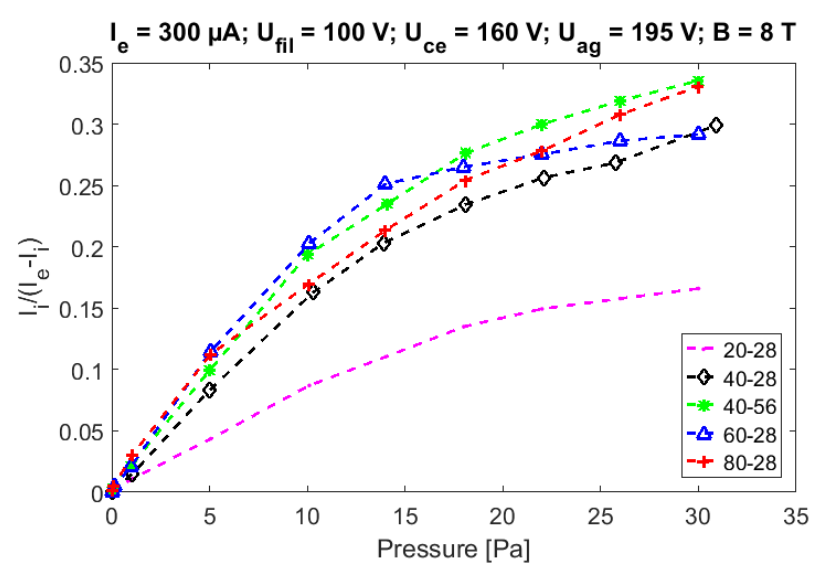

Fig. 6. Gauge output for different accelerations grids. Corresponding transparencies and number of slits are indicated in the legend.

Although all tested configurations showed a monotonic gauge output in dependence of the pressure up to at least $22 \mathrm{~Pa}$, there are considerable differences with regard to the dependence on the magnetic field strength and the sensitivity at low pressures. Very good performance was achieved for the baseline bias configuration with a grid transparency of $40 \%$ and a slit width of $0.1 \mathrm{~mm}$. The gauge output increased by roughly $8 \%$ when the pressure was raised from $18 \mathrm{~Pa}$ to $22 \mathrm{~Pa}$. In addition, the signal depended only weakly on the magnetic field above $3 \mathrm{~T}$. The sensitivity at $10^{-2} \mathrm{~Pa}$ was about $0.05 \mathrm{~Pa}^{-1}$. The results are summarized in Fig. 7 .

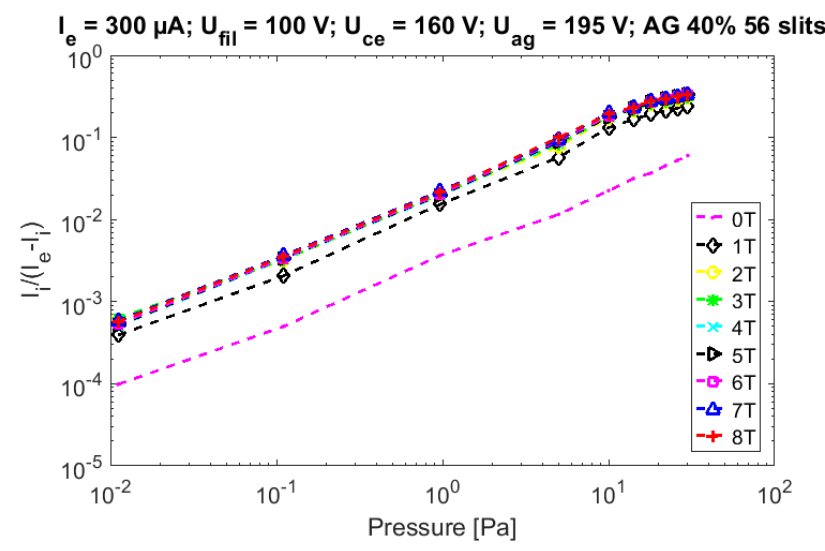

Fig. 7. Measurement performance of the gauge for the baseline bias configuration with a grid transparency of $40 \%$ and $0.1 \mathrm{~mm}$ slit width and $\mathrm{I}_{\mathrm{e}}=300 \mu \mathrm{A}$.

Assuming that the sensitivity remains unchanged for a pressure of $10^{-4} \mathrm{~Pa}$, which is the requirement for the lower pressure limit of the pressure gauges for ITER, the expected ion current is in the order of $\mathrm{nA}$. Since the pressure gauges in ITER will have to cope with long cables of up to $200 \mathrm{~m}$ length to the front-end electronics, the ion current should not go below $1 \mathrm{nA}$ to avoid problems with the data acquisition. It is also conceivable to increase the electron current significantly for low pressure operation. This action would increase the ion current level. 


\section{Conclusions}

A dedicated experiment was set up to explore the upper pressure range of an ASDEX-type pressure gauge with the aim to fulfill the ITER requirement. The starting point was an electrode bias and grid configuration that led to an optimized behavior in Monte-Carlo simulations. The gauge output was then measured as functions of pressure and magnetic flux density for different settings of the electron current, electrode bias and acceleration grid designs. The baseline configuration showed already a good performance in terms of high pressure behavior since the gauge output is monotonic up to $30 \mathrm{~Pa}$ - even higher than the ITER requirement of $20 \mathrm{~Pa}$. A higher electron current leads to a better linearity but also to a higher B-field dependence. A value of $300 \mu \mathrm{A}$ is deemed as a reasonable value. The performance was further improved by a slightly modified acceleration grid, which features a smaller width of the slits while keeping the transparency at $40 \%$. Changing the acceleration grid bias led to an even better performance at high pressure but at the expense of the dependence on the B-field and/or the linearity.

The data shows that the upper pressure limit is achievable with the current design. Slight improvements are advisable and should be implemented in the next design step. However, the repeatability of the results needs to be taken into account for a final evaluation. In an ongoing experiment, a single gauge is operated repeatedly under the same experimental conditions for several times in order to estimate the mean variation of measurements. It shall be validated with the collected data that the measurement accuracy is better than the ITER requirement of $20 \%$.

\section{Acknowledgments}

This work was partly supported by Fusion for Energy under the Specific Grant F4E-FPA-364-SG05. The views expressed in this publication are the sole responsibility of the authors and do not necessarily reflect the views of Fusion for Energy and the ITER Organization.

Neither Fusion for Energy nor any person acting on behalf of Fusion for Energy is responsible for the use, which might be made of the information in this publication.

\section{References}

[1] R. A. Pitts et al., Status and physics basis of the ITER divertor, Physica Scripta T138 (2009) 014001

[2] A. S. Kukushkin et al., Effect of neutral transport on ITER divertor performance, Nuclear Fusion 45.7 (2005) 608

[3] G. Haas and H. S. Bosch, In vessel pressure measurements in nuclear fusion experiments with ASDEX gauges, Vacuum 51 (1998) 39-46
[4] A. Scarabosio, P. Sauter and G. Haas, Modelling and optimization of ionization gauges for magnetic nuclear devices, Nuclear instruments and methods in physics research A 623 (2010) 667-671

[5] A. Scarabosio and G. Haas, Behaviour of the ASDEX pressure gauge at high neutral gas pressure and applications for ITER, AIP Conference Proceedings 988 (2008) 238. 